\title{
The emergence of hierarchy in transportation networks
}

\author{
Bhanu M. Yerra ${ }^{1}$, David M. Levinson ${ }^{2}$ \\ ${ }^{1}$ Thurston Regional Planning Council, 2424 Heritage CT SW Suite A, Olympia, WA 98502, USA \\ (e-mail: yerrab@trpc.org) \\ ${ }^{2}$ Department of Civil Engineering, University of Minnesota, 500 Pillsbury Drive SE, \\ Minneapolis, Minnesota, 55455, USA (e-mail: levin031@umn.edu)
}

Received: April 2003 / Accepted: October 2004

\begin{abstract}
A transportation network is a complex system that exhibits the properties of self-organization and emergence. Previous research in dynamics related to transportation networks focuses on traffic assignment or traffic management. This research concentrates on the dynamics of the orientation of major roads in a network and abstractly models these dynamics to understand the basic properties of transportation networks. A model is developed to capture the dynamics that leads to a hierarchical arrangement of roads for a given network structure and land use distribution. Localized investment rules, wherein revenue produced by traffic on a link is invested for that link's own development, are employed. Under reasonable parameters, these investment rules, coupled with traveler behavior, and underlying network topology result in the emergence of a hierarchical pattern. Hypothetical networks subject to certain conditions are tested with this model to explore their network properties. Though hierarchies seem to be designed by planners and engineers, the results show that they are intrinsic properties of networks. Also, the results show that roads, specific routes with continuous attributes, are emergent properties of transportation networks.
\end{abstract}

\section{Introduction}

Transportation networks possess system properties like the hierarchy of roads, accessibility, topology, geometrical features (or network properties like the ratio of nodes to links), congestion, and so on. These properties can be assumed, or we can try to determine what underlying factors lead to them. This research aims to see if, without pre-specifying a hierarchy of roads, it can be seen as an emergent property, rather than a designed one.

For a given network topology and spatial land use distribution, trips from each network node to other nodes are calculated and these trips are placed on the network. Traffic on each link pays a toll to that link and this collected revenue is invested for that link's own development, thus changing the properties of links. Using these new link properties, which change the path of least cost travel between any given origin and destination, the whole process is 
repeated until it reaches equilibrium, or it is clear that it won't reach an equilibrium.

The results show that even when land use distribution and link length are uniformly maintained, hierarchies emerge. Moreover, these hierarchies approximately replicate the rank order rule of the hierarchies in real world networks. The results also show that roads are emergent properties of networks.

The next section presents analogous theories in regional science and economics that explain formation of hierarchies, and develops the hypothesis of this research. Section 3 describes the network dynamics model. Experiments are conducted and the results are presented in Sect. 4. The paper ends with conclusions and suggestions for future research.

\section{Theoretical background}

Roads (or road links) can be classified into hierarchies according to the flow of traffic they carry. Freeways, which are at the extreme end in the hierarchical classification, carry maximum traffic while their span is relatively small when compared to the total center line miles of roads in a given geographical area. The Interstate Highway System comprises one percent of all highway miles, but carries one fourth of the U.S. vehicle miles of travel (USDOT 2000). Conversely local roads, which comprise a larger share of road length, carry little traffic, mostly from nearby users.

There are mathematical models in fields like regional science, and economics that intend to explain the formation of hierarchies of their respective subjects. Regional science explores hierarchies of places or hierarchies in the system of cities (Beckman 1958; Beckman and McPherson 1961). Economics considers formation of income categories and firm sizes (Champernowne 1953; Roy 1950). But there are few in surface transportation, as it seems to be taken for granted that planners and engineers design the hierarchies of roads. Using the example of an underdeveloped country, Taaffe et al. (1963) explain the growth of the transportation network and emergence of hierarchies of roads, however, their study does not present a mathematical model. Garrison and Marble (1965) explained the order of rail network construction in Ireland by showing that nodes would connect to the nearest large neighbor. Yamins et al. (2003) model road growth co-evolving with urban settlements from an empty space with highly simplified travel demand and road supply mechanisms.

If hierarchies and roads really emerge, rather than being designed, then there is much to learn from the disciplines mentioned above. Any explanation of the formation of hierarchies of places cannot end without mentioning the central place theory developed from works of Christaller (1933) and Lösch (1954). Christaller argues that central places emerge due to uneven distribution of facilities and transportation costs to reach these facilities. He then argues that these central places form a hierarchy. Lösch further suggested that these central places take a hexagonal shape. Krugman (1996) argues that these models don't qualify as economic models as they do not show emergence of these central places from "any decentralized process." Recently, Fujita et al. (1999) formulated a mathematical model that explains the hierarchical formation of cities from a "decentralized market process". 
Principles of complex systems have become popular among the fields that require modeling system properties from decentralized processes. There is no universally accepted definition of a complex system, however, it is generally agreed that it consists of "a large number of components or 'agents', interacting in some way such that their collective behavior is not simple combination of their individual behavior" (Newman 2001). Examples of complex systems include the economy - agents are competing firms; cities - places are agents; traffic - vehicles are agent; ecology - species are agents. Complex systems are known to exhibit properties like self-organization, emergence and chaos. Cellular Automata (CA) are commonly used to model complex systems (von Neumann 1966; Schelling 1969; Wolfram 1994, 2002). For the sake of this discussion complex systems are distinguished into two categories: One category has systems whose dynamics are modeled using networks or graphs and other has systems that are modeled using cellular automata. Literature in each of these categories is summarized below.

Watts et al. (1998) and Barabasi et al. (1999) proposed models that explain the properties and evolution of networks and graphs from localized link formations. Both works measure the formation of hierarchies of nodeconnectivity, defined for a node as number of adjacent nodes that are one link away, using power law distribution. In contrast, node-connectivity is not hierarchical in a typical road network; instead hierarchies are seen in link and node properties. It can be shown empirically that link growth and new link construction can be explained empirically by the position of the link (or potential link) in the network, its relationship with complementary (upstream and downstream) and competitive (parallel) links (Levinson and Karamalaputi 2003a,b). However, research into transportation networks can greatly benefit the Barabasi et al.'s (1999) concept of preferential attachment, which is akin to the concept of the "rich get richer", as the source of hierarchies.

Epstein and Axtell (1996) modeled social processes using agent based cellular automata. They modeled a "Sugarscape" - a landscape of resources and placed interacting agents - rational decision makers - on it to simulate social processes. They showed the emergence of social wealth and age distributions (which can be considered as social hierarchies) from localized interactions of agents. They also modeled the dynamics of trade networks, credit networks and disease transmission networks using agent interactions (but did not model transportation networks). Nagel and Schreckenberg (1992) and Schadschneider and Schreckenberg (1993) have used cellular automata concepts to model traffic but did not model transportation network dynamics.

In the above mentioned complex system studies, irrespective of how a system is modeled, simple local interaction rules are shown as sufficient to model the self-organizing properties of the system. In all those studies, the self-organizing behavior is measured using power law distributions. Measuring self-organization in emerging systems using power law distribution is well explained by Bak (1996).

\section{Network dynamics model}

In this research a network dynamics model is developed that brings together all the relevant transportation models to simulate network growth. An 


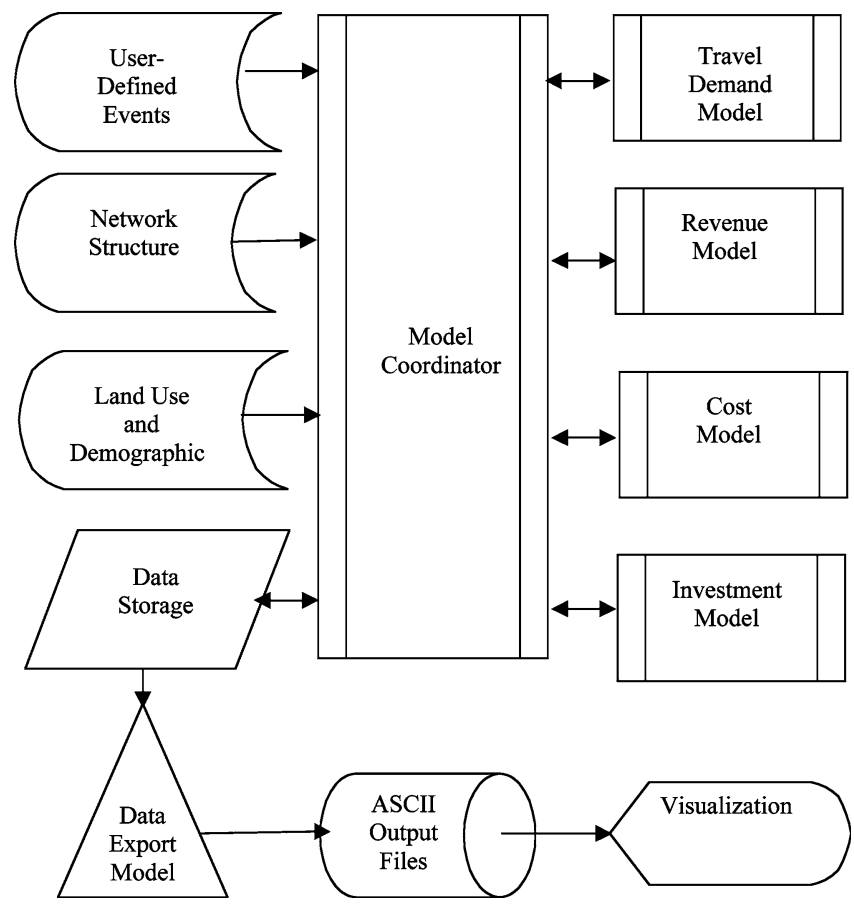

Fig. 1. Overview of modeling process

overview of transportation models and their interconnectivity is shown in Fig. 1. Network structure, land use and demographic information, and userdefined events are exogenous inputs. In formulating the dynamics from a complex systems perspective, the network nodes, road links and travelers become agents. A schematic representation of the network structure and land use is show in Fig. 2. The network structure and land use data are used by the travel demand model to calculate traffic flows on links. Traffic is assigned to links along the path with the least generalized cost of travel. A revenue model determines the toll the traffic must pay for using the road depending on speed, flow and length of the link. A cost model determines how much it costs to maintain the present level of service. Depending on revenue and cost, the investment model determines the link properties (speed) for the next time step. This process is repeated until an equilibrium of link speeds has been reached, or it is clear that no equilibrium can be reached. The output files produced are then exported to a visualization tool and the dynamics are viewed in a movielike fashion. The details of mathematical formulations of these transportation models are presented in the following sub-sections.

\section{Network structure}

The transportation network is represented as a directed graph that connects nodes with directional arcs (links). The directed graph is defined as: $G=$ $\{N, A\}$ where $N$ is a set of sequentially numbered nodes and $A$ is a set of 


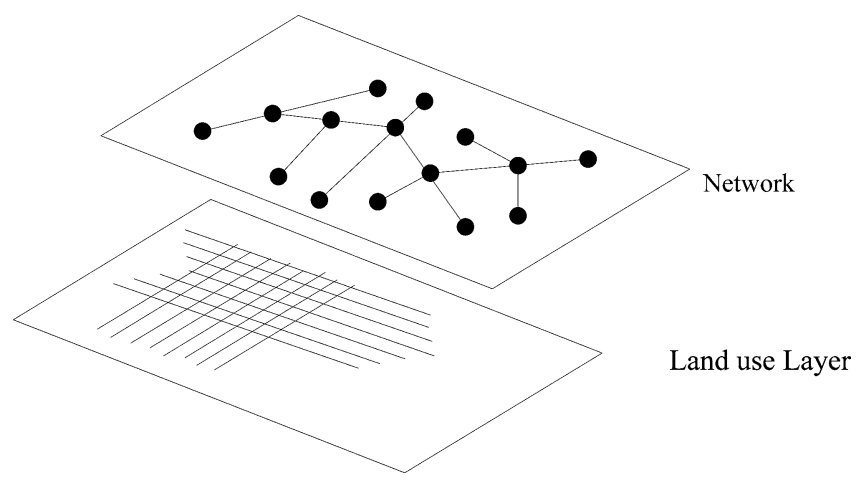

Fig. 2. The network and land use layer

sequentially numbered directed arcs. An arc ' $a$ ' connected from origin node $\mathrm{m}$ to destination node $\mathrm{n}$ is represented as $m \rightarrow n$. Let $R$ denote a set of origin nodes and $S$ denote a set of destination nodes. Note that in this network $R=S=N$, i.e. each node acts as both origin and destination. Let $x_{n}$ and $y_{n}$ represent the $x$ and $y$ coordinates of node $n \in N$ in Cartesian coordinate system. Let $v_{a}^{0}$ be the initial speed on link $a \in A$. Let $l_{a}$ be the length of the link $a$.

\section{Travel demand model}

Trip generation model. The geographical area under consideration is divided into cells (e.g., city blocks) and the land use is distributed among these cells. After reading the network, the trip generation model reads the size of the underlying land use layer in terms of number of cells and assigns the cells to the nearest network node. The land use layer is assumed to be static throughout the simulation. The land use layer is modeled as a square. Each cell is given two properties that represent the trips attracted $\left(g_{z}\right)$ and trips generated $\left(h_{z}\right)$ from that cell $(z)$. Using the cell properties, trips produced at and trips attracted to a network node can be calculated by summing up the trips produced at and trips attracted to all cells that are nearest to that node. Let $\mathrm{g}_{n}$ and $\mathrm{h}_{n}$ be trips produced at and trips attracted to network node $n$.

Shortest path algorithm. Let $t_{a}^{i}$ represent the generalized cost on link $a$ for iteration $i$. This is calculated as the linear combination of link travel time and the toll ( $\tau$ as shown in Eq. (1)), assuming the weights to dimensionally balance the equation are ones.

$$
t_{a}^{i}=\frac{l_{a}}{v_{a}^{i}}+\tau\left(l_{a}\right)^{\rho_{1}} \quad \forall a \in A
$$

where, $\rho_{1}$ is a coefficient.

From each node in the network, a least cost path to every other node is calculated using Dijkstra's Algorithm (Chachra et al. 1979). Let $K_{\mathrm{rs}}$ represents a set of arcs along the least cost path from origin $r$ to destination $s$ for 
iteration $i$. Let $t_{r s}^{i}$ represent the travel cost from origin $r$ to destination $s$ along the least cost path for iteration $i$. Then the relationship between $K_{r s}^{i}$ and $t_{r s}^{i}$ is:

$$
t_{r s}^{i}=\sum_{a \in A} t_{a}^{i} \delta_{a, r s}^{i} \quad \forall r \in R, \quad \forall s \in S
$$

where $\delta_{a, r s}^{i}$ is a dummy variable equal to 1 if arc $a$ belongs to $K_{r s}^{i}, 0$ otherwise.

Trip distribution. With the trip generation values and travel costs, a trip table (Origin Destination (OD) matrix) is computed using a gravity model (Hutchinson 1974; Haynes and Fotheringham 1984). Let $q_{r s}$ be the number of trips from origin node $r$ that are ending at destination node $s$. The gravity model indicates that $q_{r s}$ is directly proportional to trips produced from origin node $r\left(g_{r}\right)$ and trips attracted to destination node $s\left(h_{s}\right)$ and is inversely proportional to the generalized cost of travel from origin node $r$ to destination node $s$ as shown below.

$$
q_{r s} \propto \frac{g_{r} h_{s}}{d(r, s)} \quad \forall r \in R, \quad \forall s \in S
$$

where, $d(r, s)$ is called the friction factor. The friction factor function used in the gravity model is a negative exponential as shown below:

$$
d(r, s)=e^{-\gamma \cdot t_{r s}}
$$

where, $\gamma$ is a coefficient that represents commuters disutility as costs of travel rise.

The resulting OD matrix is also incorporates the reverse trips from an origin to a destination to account for the evening traffic returning home. The resulting OD matrix $q_{r s}^{*}$ is calculated as shown below.

$$
q_{r s}^{*}=q_{r s}+q_{s r} \quad \forall r \in R \quad \forall s \in S
$$

Traffic assignment. Using the OD matrix and shortest routes, traffic is assigned to each link. Flow $\left(f_{a}\right)$ on each link is the sum of all the flows of paths between any origin and destination that passes through that link.

$$
f_{a}=\sum_{r s} q_{r s}^{*} \delta_{a, r s} \quad \forall a \in A
$$

\section{Revenue model}

This is a link-based model that calculates revenue for each link. Revenue is calculated by multiplying the toll and flow. Therefore the higher the flow on the link, the higher is the revenue. This model assumes that revenue is only collected by vehicle toll.

$$
E_{a}=\left(\tau \cdot\left(l_{a}\right)^{\rho_{1}}\right) \cdot \psi \cdot f_{a} \quad \forall a \in A
$$

where $\psi$ is a model parameter to balance the equation dimensionally and to convert the daily flow to annual. 


\section{Cost model}

This model calculates the cost to keep a link in its present usable condition depending on the flow, speed, and length.

$$
C_{a}=\mu \cdot\left(l_{a}\right)^{\alpha_{1}}\left(f_{a}\right)^{\alpha_{2}}\left(v_{a}\right)^{\alpha_{3}} \quad \forall a \in A
$$

where, $C_{a}^{i}$ is the cost of maintaining the road at its present condition, $\mu$ is the (annual) unit cost of maintenance for a link times the conversion factor to dimensionally balance the equation, $\alpha_{1}, \alpha_{2}, \alpha_{3}$ are coefficients indicating economies or diseconomies of scale

\section{Investment model}

Depending on the available revenue and maintenance costs this model changes the speed of every link at the end of each time step as shown in Eq. (9). If the revenue generated by a link is insufficient to meet its maintenance requirements i.e. $E_{a}<C_{\mathrm{a}}$, its speed drops. If the link has revenue remaining after maintenance, it invests that remaining amount in capital improvements, increasing its speed. This, along with a shortest path algorithm, embeds the "rich get richer" logic of link expansion. A major assumption in this model is that a link uses all the available revenue in a time step without saving for the next time step.

$$
v_{a}^{i+1}=v_{a}^{i}\left(E_{a}^{i} / C_{a}^{i}\right)^{\beta} \quad \forall a \in A
$$

where, $\beta$ is speed improvement coefficient.

With the new speed on the links the travel time changes and the whole process from the travel demand model is iterated to grow the transportation network until the network reaches equilibrium, or it is clear that it won't.

\section{Experiments and results}

The network dynamics model presented in the previous section provides a platform to conduct experiments on transportation networks to study their properties and dynamics. Several experiments are conducted and the results are presented in this section.

\section{Base case}

A base case is chosen and variations are made to this case to study how these variations affect the dynamics and the resulting hierarchies. The base case consists of an evenly spaced grid network in the form of a square with each link having the same initial speed. Each land use cell produces and attracts the same number of trips. The network structure and land use properties are chosen this way to eliminate network asymmetries as a confounding factor. Speeds on links running in the opposite direction between the same nodes are averaged in this case. Since Dijkstra's algorithm does not list all possible shortest paths between any two nodes, symmetry conditions are externally 
Table 1. Model parameters and values used for experiments in paper

\begin{tabular}{|c|c|c|c|c|c|}
\hline \multirow[t]{2}{*}{ Variable } & \multirow[t]{2}{*}{ Description } & \multirow{2}{*}{$\begin{array}{l}\text { Base } \\
\text { case }\end{array}$} & \multirow{2}{*}{$\begin{array}{l}\text { Experiment } \\
\text { A }\end{array}$} & \multicolumn{2}{|c|}{ Experiment B } \\
\hline & & & & Case-1 & Case-2 \\
\hline$v_{a}^{0}$ & Initial speed (integer) & 1 & $1-5$ & 1 & $1-5$ \\
\hline$g_{z}, h_{\mathrm{z}}$ & Land use properties of cell $z$ & 10 & 10 & $10-15$ & $10-15$ \\
\hline$\gamma$ & $\begin{array}{l}\text { Coefficient in Eq. (4) trip } \\
\text { distribution model }\end{array}$ & 0.01 & 0.01 & 0.01 & 0.01 \\
\hline$\rho_{1}$ & Length power in revenue model & 1.0 & 1.0 & 1.0 & 1.0 \\
\hline$\tau$ & Tax rate in Eq. (7) revenue model & 1.0 & 1.0 & 1.0 & 1.0 \\
\hline$\psi$ & Revenue model parameter in Eq. (7) & 365 & 365 & 365 & 365 \\
\hline$\mu$ & Unit cost in Eq. (8) cost model & 365 & 365 & 365 & 365 \\
\hline$\alpha_{1}$ & Length power in Eq (8) cost model & 1.0 & 1.0 & 1.0 & 1.0 \\
\hline$\alpha_{2}$ & Flow power in Eq. (8) cost model & 0.75 & 0.75 & 0.75 & 0.75 \\
\hline$\alpha_{3}$ & Speed power in Eq. (8) cost model & 0.75 & 0.75 & 0.75 & 0.75 \\
\hline$\beta$ & $\begin{array}{l}\text { Coefficient in Eq. (9) investment } \\
\text { model }\end{array}$ & 1.0 & 1.0 & 1.0 & 1.0 \\
\hline
\end{tabular}

applied. Table 1 shows the parameters used in base case and other experiments. The results for a 10 node by 10 node network are shown in Fig. 3 .

Figure $3 \mathrm{~b}$ shows the spatial distribution of speed for the network at equilibrium. The entire range of link speeds is divided into 4 equal intervals and these interval categories are used in drawing the figure with the line thickness and color representing the speed category. The spatial distribution of speeds depends on the parameters used in the model. Results clearly show that hierarchies and roads emerge from a localized link-based investment process. Despite controlling the land use and link length, we believe hierarchies are emerging because of the travel behavior induced by the presence of a boundary. Travel demand along the edges is inward while trips are evenly distributed along all possible directions in the middle of the area.

It can be argued that if edges are eliminated in the base case by carefully molding the geography into a torus while maintaining uniform land use and

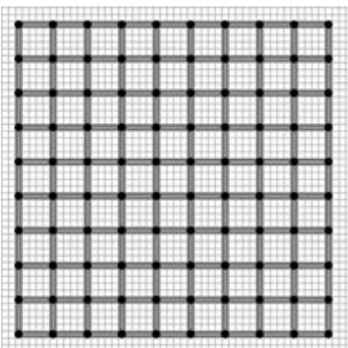

a

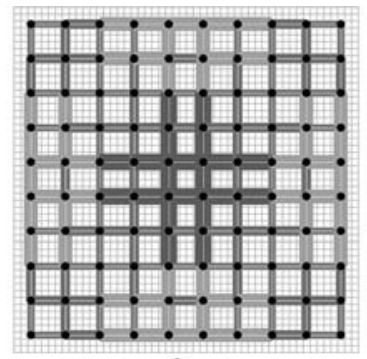

b

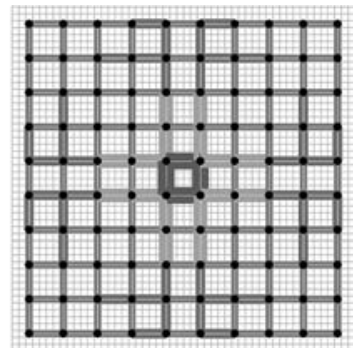

C

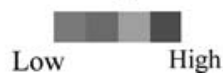

Fig. 3. (a) Spatial distribution of speed for the initial network; (b) Spatial distribution of speed for the network at equilibrium reached after 8 iterations; (c) Spatial distribution of traffic flow for the network at equilibrium. The color and thickness of the link shows its relative speed or traffic flow 
uniform spacing between links and adding new links to connect the edges, then any link from the resulting network becomes indistinguishable from any other link irrespective of its orientation along the meridian or longitudinal axis. This is the ideal case that produces no hierarchies or identifiable roads. Easing any conditions in this "ideal torus" case will result in hierarchies. In other words, the edges of the network are the force creating the hierarchy the greater utility of central links for traffic increases their flow, and thus their speed. The investment model is also an important factor leading to the formation of hierarchies, which parallels Barabasi et al.'s (1999) concept of preferential attachment leading to formation of hierarchies.

Having examined the reasons for formation of hierarchies it is now time to reason for the converging solution. For a given static land use, the economies of scale in the cost function associated with traffic flow $\left(\alpha_{2}<1\right)$ along with increasing cost for higher link speeds $\left(\alpha_{3}>0\right)$ drives the system to an equilibrium. Diseconomies of scale in the cost function with respect to traffic flows result in an oscillating equilibrium.

\section{Experiment $A$}

Experiment $\mathrm{A}$ is similar to the base case except for randomly distributing the initial link speeds between 1 and 5. The network is evolved until an equilibrium is reached. Since random distribution of speeds makes the results stochastic, 20 such cases are performed and the average of the results are taken. A typical solution is shown in Fig. 4.

Emergence of hierarchies and roads are clearly seen in this case. Random distribution of initial speeds produces non-symmetrically oriented roads with most of the faster links concentrated in the center of the geography. Belt or ring roads are common. Figure $4 \mathrm{c}$ shows the spatial distribution of traffic flow. Notice that there are fewer links that carry more traffic (thicker links in red) and many links that carry less traffic (thinner links in green), resembling a rank order rule. To investigate this rank order behavior of traffic flows of the network at equilibrium, results are compared with flow distribution of the

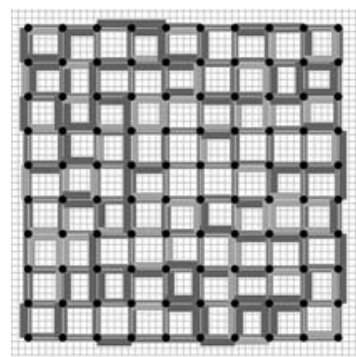

a

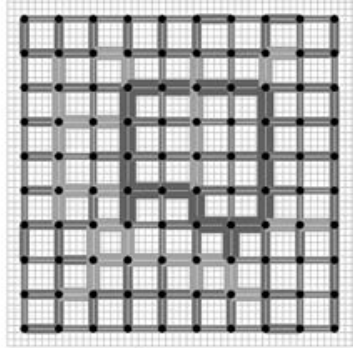

b

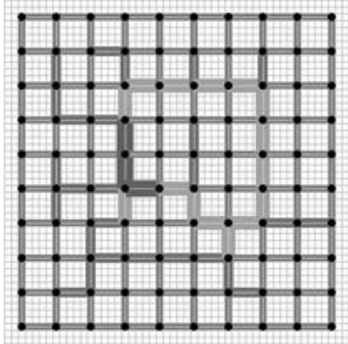

C

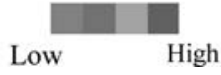

Fig. 4. (a) Spatial distribution of initial speeds; (b) Spatial distribution of speeds for the network at equilibrium; (c) Spatial distribution of traffic flow for the network at equilibrium. The color and thickness of the link shows its relative speed or traffic flow 


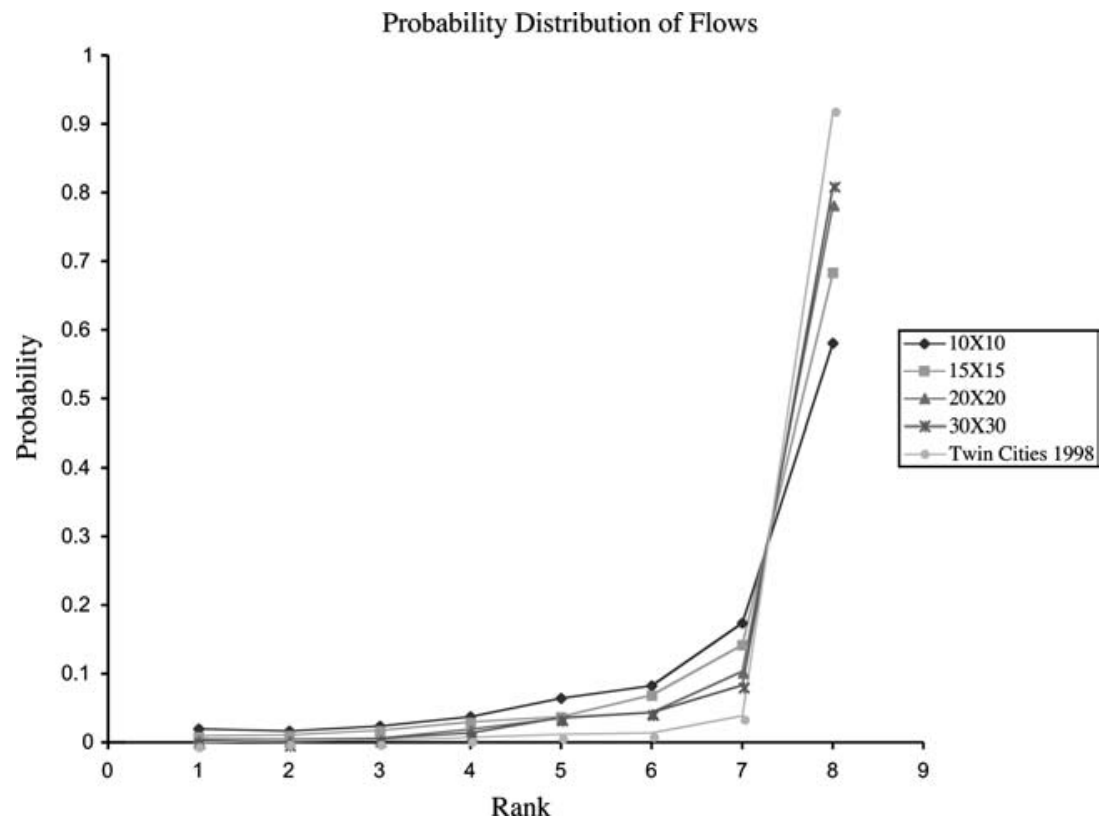

Fig. 5. Comparison of traffic flow distribution of higher order networks with 1998 Twin Cities AADT distribution, Experiment A

Minneapolis and St. Paul (Twin Cities) network. Figure 5 compares the probability distribution of traffic flow for higher order networks for this case with probability distribution of 1998 Average Annual Daily Traffic (AADT) on links in the Twin Cities, where such counts are taken.

For this graph, the entire range of flow distribution is divided into 8 intervals of equal size and the number of links falling in each interval is counted. These counts are divided by the total number of links in the network to get probabilities. These intervals are given a rank; the lower the flow range in the interval, the higher is the rank.

Notice in the graph as the size of the network increases, the probability distribution of the grid networks tend to get closer to the Twin Cities flow distribution. This graph concretely establishes that it is possible to replicate the global properties of real transportation networks by growing the network system using localized investment rules.

\section{Experiment $B$}

Experiment B is similar to the base case except for the treatment of initial speeds and land use characteristics. Land use characteristics of the cells are randomly distributed across the landscape between 10 and 15 trips. In this case, unlike the previous case, trips produced and trips attracted from a land use cell need not be the same. Link speeds in this model are dealt in two ways; firstly (experiment B1), initial speeds are assumed to be same for each link with magnitude 1, same as base case. Secondly (experiment B2), 


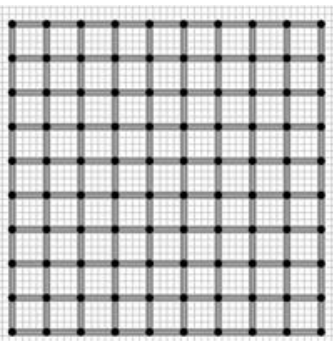

a

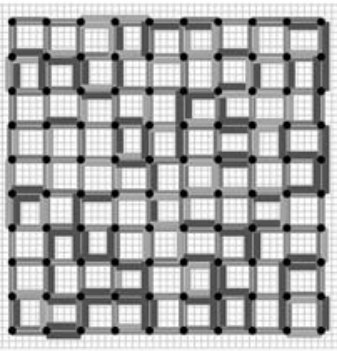

d

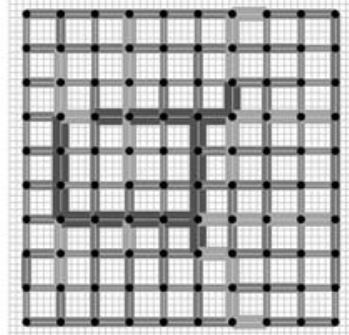

b

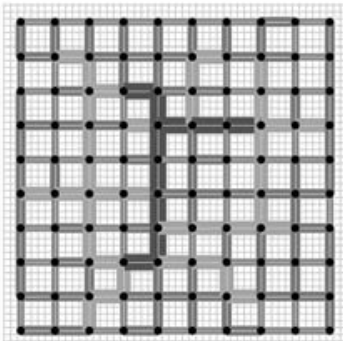

e

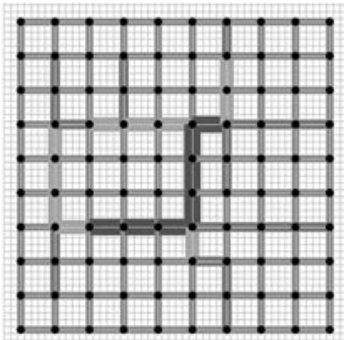

C

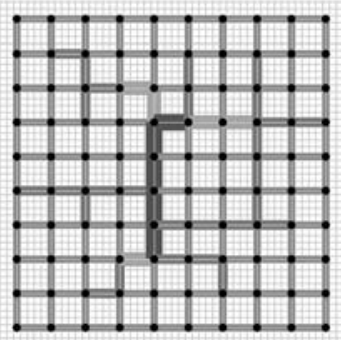

f

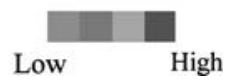

Fig. 6. (a) and (d) Spatial distribution of initial speed for experiments B1 and B2 respectively; (b) and (e) Spatial distribution of speeds for the network after reaching equilibrium; (c) and (f) Spatial distribution of traffic flows for the network after reaching equilibrium. The color and thickness of the link shows its relative speed or flow

initial speeds are randomly distributed between 1 and 5 as it was done experiment A. Typical solutions for experiment B1 and B2 are shown in Fig. 6.

Notice the similarity of this experiment with the previous case. We believe the differences in land use distribution (and initial speeds in case of experiment $2 b$ ) and the boundaries are responsible for the hierarchies in this case. Similar to the previous case, a rank order rule is observed in this experiment. The probability distribution of flows for this case are compared with 1998 AADT distribution of the Twin Cities in the Fig. 7.

Notice that as the network size increases the behavior of traffic flow distribution is approaches the behavior of the Twin Cities, similar to the observation in experiment A.

\section{Conclusions}

This paper presents a transportation network dynamics model that includes localized revenue and investment models. This model can be considered as a "bottom-up" approach of modeling the emergence of hierarchies and roads, which are observed in several experiments. Therefore, it is possible to grow hierarchies and roads in a transportation network using decentralized investment rules. The network, no matter how random the initial speed 


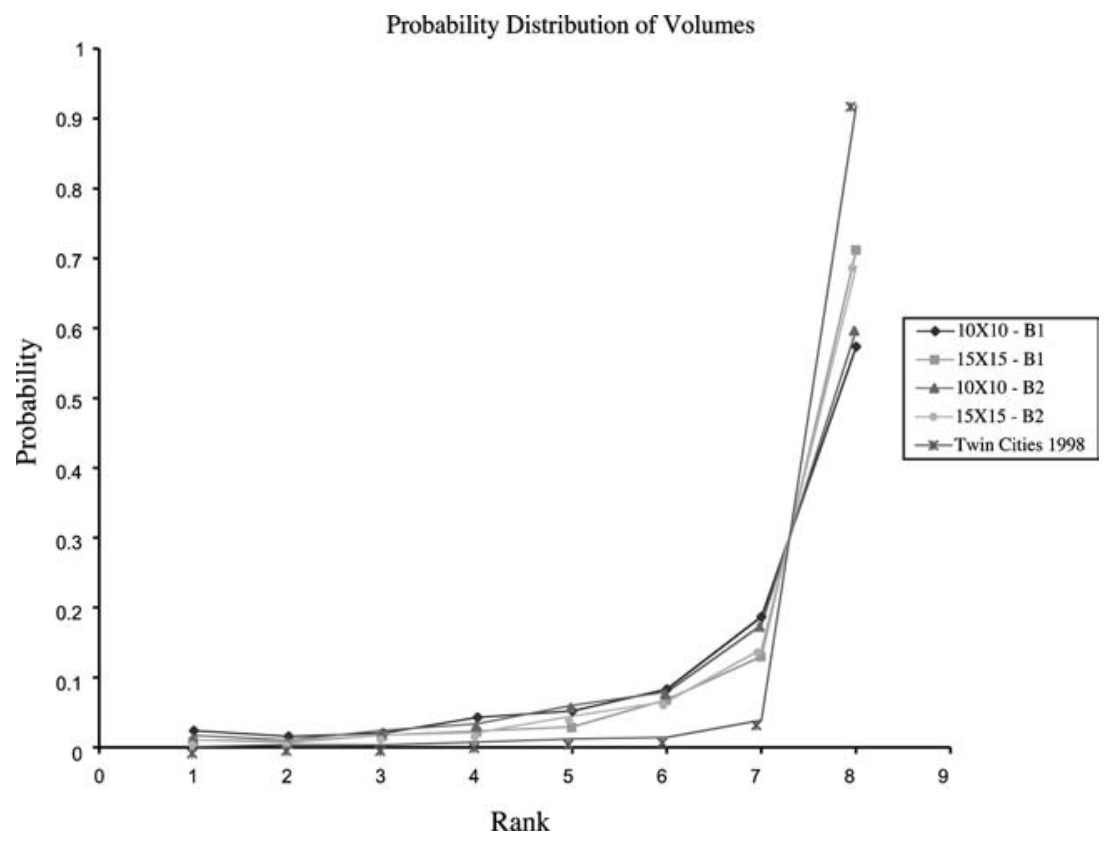

Fig. 7. Comparison of traffic flow distribution of higher order networks with 1998 Twin Cities AADT distribution, Experiment B

distribution, when grown subject to localized investment rules produces order by self-organization.

If one looks at the complexity and bureaucracy involved in transportation infrastructure investment, one might conclude that it is impossible to model transportation network dynamics endogenously. But this research has shown that simple localized investment rules can be used to reflect the overall system properties. In fact, it is not the results that are most striking, but the simplicity of the investment rules in mimicking the system properties.

A new way of modeling and testing network dynamics is created by this research, which opens numerous opportunities of future research that can contribute immensely to our understanding of network dynamics. Research exploring different possibilities of investment rules that can not only reflect the global properties of networks, but also the network structure itself, can be considered. A realistic network can be used in these experiments instead of hypothetical grid networks and the coefficients can be estimated. More sophisticated travel demand models can be used. The cost model represented here can be made more realistic by introducing a construction cost function. A revenue sharing model - allowing links to share their revenue if they have excess - can be introduced and may produce much richer and realistic dynamics. Further examination of the rank-order rule is warranted. More research and a paper with application of this model to a real world network as a demonstration will encourage engineers and planners to adopt these kinds of models. 


\section{References}

Bak P (1996) How nature works: The science of self-organized criticality. Copernicus (SpringerVerlag), New York, NY

Barabasi A-L, Albert R (1999) Emergence of scaling in random networks. Science 286: 509-512

Beckmann MJ (1958) City hierarchies and the distribution of city sizes. Economic Development and Cultural Change 6: 243-248

Beckmann MJ, McPherson J (1970) City size distributions in a central place hierarchy: An alternative approach. Journal of Regional Science 10: 25-33

Chachra V, Ghare PM, Moore JM (1979) Applications of Graph Theory Algorithms. North Holland, New York

Champernowne DG (1953) A model of income distribution. The Economic Journal 63: 318-351

Christaller W (1933) Central places in Southern Germany. Fischer (English translation by C. W. Baskin, London: Prentice Hall, 1966) Jena, Germany

Epstein JM, Axtell R (1996) Growing artificial societies. MIT Press, Cambridge, MA

Fujita M, Krugman P, Venables AJ (1999) The spatial economy: cities, regions, and international trade. MIT Press, Cambridge, MA

Garrison WL, Marble DF (1965) A prolegomenon to the forecasting of transportation development. Office of Technical Services, United States Department of Commerce, United States Army Aviation Material Labs Technical Report

Haynes KE, Fotheringham AS (1984) Gravity and spatial interaction models. Sage Publications, Beverly Hills, CA

Hutchinson BG (1974) Principles of urban transportation systems planning. McGraw-Hill, New York

Krugman P (1996) The self-organizing economy. Blackwell, New York

Levinson D, Karamalaputi R (2003) Predicting the construction of new highway links. Journal of Transportation and Statistics 6(2/3): 81-89

Levinson D, Karamalaputi R (2003) Induced supply: a model of highway network expansion at the microscopic level. Journal of Transport Economics and Policy 37(3): 297-318

Lösch A (1954) The economics of locations. Yale University Press, New Haven

Nagel K, Schreckenberg A (1992) A cellular automata model for freeway traffic. Journal de Physique 2: 2221-2229

Newman MEJ (2002) The structure and function of networks. Computer Physics Communications 147: 40-45

Roy AD (1950) The distribution of earnings and individual output. Economic Journal 60: 489505

Schadschneider A, Schreckenberg M (1993) Cellular automata models and traffic flow. Journal of Physics A: Mathematical and General 26: 679-683

Schelling TC (1969) Models of segregation. American Economic Review, Papers and Proceedings 59(2): 488-493

Taaffe EJ, Morrill RL, Gould PR (1963) Transport expansion in underdeveloped countries: a comparative analysis. Geographical Review 53(4): 503-529

U.S. Department of Transportation, Bureau of Transportation Statistics (2000) The changing face of transportation. BTS00-007. Washington, DC

Watts DJ, Strogatz SH (1998) Collective Dynamics of 'small-world' networks. Nature 393: 440442

Von Neumann J (1966) Theory of self-reproducing automata. In: Burks AW (ed). University of Illinois Press

Wolfram S (1994) Cellular automata and complexity. Addison-Wesley, Reading, MA

Wolfram S (2002) A new kind of science. Wolfram Media, Champaign, IL

Yamins D, Rasmussen S, Fogel D (2003) Growing urban roads. Networks and Spatial Economics 3: 69-85 